\title{
Functional improvement of porcine neonatal pancreatic cell clusters via conformal encapsulation using an air-driven encapsulator
}

\author{
Sol Ji Park ${ }^{1 *}$, Soojeong Shin ${ }^{2 *}$, Ok Jae Koo ${ }^{1,3}$, \\ Joon Ho Moon ${ }^{1}$, Goo Jang ${ }^{1}$, Curie Ahn ${ }^{3,4,5}$, \\ Byeong Chun Lee $\mathrm{e}^{1,6}$ and Young Je Yoo ${ }^{2,6}$ \\ ${ }^{1}$ Department of Theriogenology and Biotechnology \\ College of Veterinary Medicine \\ Research Institute for Veterinary Science \\ ${ }^{2}$ School of Chemical and Biological Engineering \\ College of Engineering \\ Seoul National University \\ Seoul 151-742, Korea \\ ${ }^{3}$ Transplantation Research Institute \\ ${ }^{4}$ Department of Medicine \\ College of Medicine \\ Seoul National University \\ Seoul 110-744, Korea \\ ${ }^{5}$ Transplantation Center \\ Seoul National University Hospital \\ Seoul 110-744, Korea \\ ${ }^{6}$ Corresponding authors: Tel, 82-2-880-1269; \\ Fax, 82-2-873-1269; E-mail, bclee@ snu.ac.kr (B.C.L.) \\ Tel, 82-2-880-7411; Fax, 82-2-887-1659; \\ E-mail, yjyoo@ snu.ac.kr (Y.J.Y.) \\ *These authors contributed equally to this work. \\ http://dx.doi.org/10.3858/emm.2012.44.1.001
}

Accepted 10 October 2011

Available Online 19 October 2011

Abbreviations: DW, distilled water; ECM, extracellular matrix; IBMX, 3-isobutyl-1-methylxanthine; NPCC, neonatal pancreatic cell clusters; $\mathrm{SI}$, stimulation index

\begin{abstract}
Transplantation of islet cells into diabetic patients is a promising therapy, provided that the islet cells are able to evade host immune rejection. With improved islet viability, this strategy may effectively reverse diabetes. We applied $2 \%$ calcium alginate to generate small and large capsules to encapsulate porcine neonatal pancreatic cell clusters (NPCCs) using an air-driven encapsulator. After encapsulation, the viability was assessed at 1, 4, 7, 14 and 28 days and secretion of functional insulin in response to glucose stimulation were tested at days 14 and 28. Selective permeability of the
\end{abstract}

small alginate capsules was confirmed using various sizes of isothiocyanate-labeled dextran (FITC-dextran). Encapsulation of NPCCs was performed without islet protrusion in the small and large capsules. The viability of NPCCs in all experimental groups was greater than $90 \%$ at day 1 and then gradually decreased after day 7. The NPCCs encapsulated in large capsules showed significantly lower viability $(79.50 \pm 2.88 \%)$ than that of naïve NPCCs and NPCCs in small capsule $(86.83 \pm 2.32 \%, 87.67 \pm 2.07 \%$, respectively) at day 7 . The viability of naïve NPCCs decreased rapidly at day $14(75.67 \pm 1.75 \%)$, whereas the NPCCs encapsulated in small capsules maintained $(82.0 \pm 2.19 \%)$. After 14 and 28 days NPCCs' function in small capsules $(2.67$ \pm 0.09 and $2.13 \pm 0.09$ ) was conserved better compared to that of naïve NPCCs $(2.04 \pm 0.25$ and $1.53 \pm$ 0.32 , respectively) and NPCCs in large capsules (2.04 \pm 0.34 and $1.13 \pm 0.10$, respectively), as assessed by a stimulation index. The small capsules also demonstrated selective permeability. With this encapsulation technique, small capsules improved the viability and insulin secretion of NPCCs without islet protrusion.

Keywords: alginic acid; diabetes mellitus, type 1 ; insulin-secreting cells; islets of Langerhans transplantation; tissue therapy

\section{Introduction}

Diabetes mellitus type 1 is one of the most common endocrine diseases and is characterized by an absolute insulin deficiency. Insulin injection therapy has been known as the most effective way for the cure and has improved the quality of life for diabetes mellitus patients; however, chronic complications of the eyes, kidneys, cardiovascular system or hypoglycemic shock cannot be avoided. The only replacement therapy to improve the metabolic control in diabetes mellitus patients other than conventional insulin therapy is the transplantation of insulin-producing cells, pancreatic islets or whole pancreas. However, there are major obstacles for both pancreas and islet transplantation, including the requirement of immunosuppressive drugs to prevent graft rejection and shortage of organ donors. 
Cell encapsulation strategies have been suggested as a solution to both the problems associated with immunosuppression and organ shortage problems. These technologies are based on the principle that foreign cells are protected against the host immune systems, including antibodies and cytotoxic cells, by sequestering them within an artificial membrane. Immunoprotection by encapsulation enables allo- or xeno-transplantation without immunosuppressive drugs and provides the perspective of animal sources like pig. The microencapsulation of cells in alginate-based capsules has been described by Lim and Sun (1980) and is the most commonly applied procedure for immunoisolation. Despite the simplicity of microencapsulation and the urgent need for alternatives to immunosuppressives in transplantation, progress in this field remains slow and inefficient to meet high expectations. Major challenges are stilled encountered, such as decreased metabolites, $\mathrm{O}_{2}$ transfer associated with the thick membrane of large capsules $(>700 \mu \mathrm{m})$ and total volume of microencapsulated islet used for transplantation. Therefore, the need exists to develop islet encapsulation strategies that minimize transplant volume and maximize metabolite transfer.

This critical demand has led to the design and application of novel concepts regarding cell encapsulation for transplantation. Here, we demonstrate the formation of conformal microcapsules (i.e., small capsules) using an air-driven encapsulator. We compared and analyzed in the view of pancreatic cell viability and insulin-release function in response to glucose stimulation.

\section{Results}

\section{Optimization of encapsulation}

Test for capsule size optimization was performed to

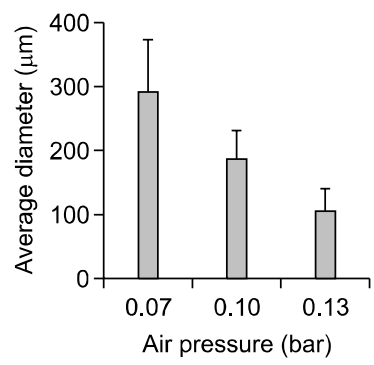

Figure 1. Average diameter of alginate capsules produced by aerodynamic encapsulator with $135 \mathrm{ml} / \mathrm{h}$ of $2 \%$ (w/v) alginate flow rate through $500 \mu \mathrm{m}$ nozzle $(\mathrm{n}>160)$. Under the condition of 0.10 bar of air pressure, average diameter was $184.0( \pm 46.1) \mu \mathrm{m}$, overlapped range of diameter of NPCCs. minimize dead space filled with calcium alginate only. Capsule size was increased by decrement of air pressure with fixed flow rate of alginate solution. Capsule produced under the condition of alginate flow rate of $135 \mathrm{ml} / \mathrm{h}$ and air pressure of 0.10 bar had closest size with islets, as shown in Figure 1.

\section{Encapsulation of NPCC using an air-driven encapsulator}

The encapsulation of NPCCs in small and large capsules was performed successfully. Moreover, the small capsules prepared using an air-driven encapsulator showed no NPCC protrusion (Figure 2B). The NPCCs in small capsules had a thin alginate layer, which did not exceed $200 \mu \mathrm{m}$. Islet cells were dissociated from naïve NPCCs (Figure $2 \mathrm{~A}$ ); however, the encapsulated NPCCs were not because encapsulated NPCCs were mechanically supported (Figures 2B and 2C).

\section{In vitro assessments of encapsulated NPCC}

We compared the naïve NPCCs and NPCCs in the small and large capsules according to their viability and insulin release. The NPCC viability was $>90 \%$ at day 1 in all experimental groups and then decreased gradually after week 1 . The NPCCs in large capsules showed significantly low viability

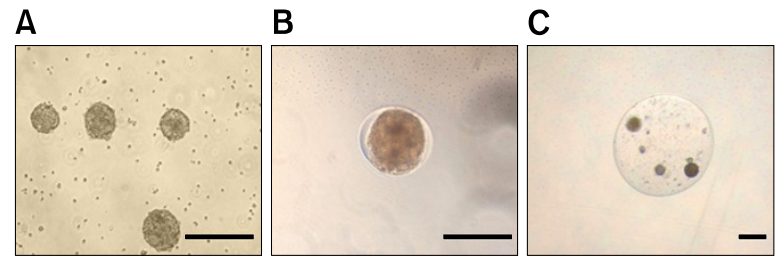

Figure 2. Microscopy images of naïve NPCCs (A), NPCCs encapsulated in a small capsule (B) and NPCCs encapsulated in a large capsule (C). The magnifications are $100 \times, 100 \times$ and $40 \times$, respectively and the scale bars represent $200 \mu \mathrm{m}$.

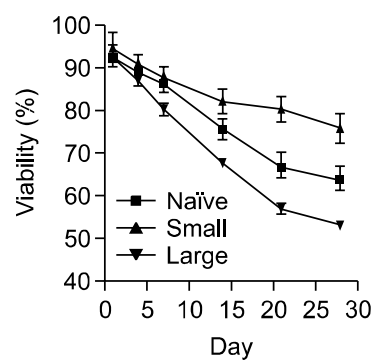

Figure 3. Viability of the NPCCs encapsulated in small and large capsules. Cell viability was evaluated at days $1,4,7,14,21$ and 28 throughout a 28-day study. The viability values are displayed as the average from six performed trials $(P<0.05)$. 
A

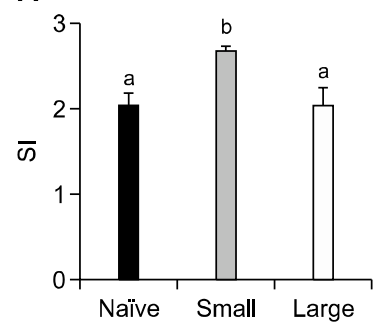

B

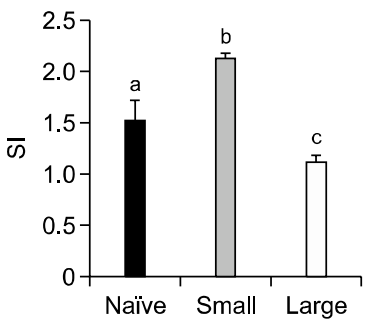

Figure 4. Glucose-stimulated insulin release from small and large encapsulated NPCCs. As a control, the assay was performed on the naïve islets. The insulin amounts that were secreted from the NPCCs in response to glucose concentration changes ranging from 2.8 to $20 \mathrm{mM}$ were determined by electrochemiluminescence immunoassay analyses. The assays were replicated six times. The data was collected at day 14 (A) and day 28 (B). The values for the different superscripts are significantly different $(P<0.05)$.

$(79.50 \pm 2.88 \%)$ than that of the naïve NPCCs and NPCCs in small capsules $(86.83 \pm 2.32 \%, 87.67$ $\pm 2.07 \%$, respectively) at day 7 . The viability of naïve NPCCs decreased rapidly at day 14 (75.67 $\pm 1.75 \%$ ), whereas the NPCCs encapsulated in small capsules maintained good viability $(82.0 \pm$ $2.19 \%$ ) (Figure 3).

The insulin secretion of the islets encapsulated within calcium alginate microcapsules was investigated and compared with that of naïve islets by measuring the glucose-stimulated insulin secretion with 2.8 and $20 \mathrm{mM}$ glucose (Figure 4). In terms of insulin secretion, the encapsulated islets secreted insulin in response to glucose in static culture medium. The NPCCs encapsulated within small capsules $(2.67 \pm 0.09,2.13 \pm 0.09)$ showed a significantly higher $\mathrm{SI}$ than that of the naïve NPCCs $(2.04 \pm 0.25,1.53 \pm 0.32)$ and NPCCs in large capsules $(2.04 \pm 0.34,1.13 \pm 0.10)$ in response to glucose stimulation at days 14 and 28 .

\section{Permeability studies}

We also confirmed that the small capsules induced higher viability and insulin release and allowed the penetration of low molecular weight molecules, such as glucose and insulin, while blocking high molecular weight molecules, including antibodies. As a result, the small capsules ranged from 100 to $200 \mu \mathrm{m}$ and successfully blocked the penetration of $150 \mathrm{kDa}$ dextran, while allowing penetration of $20 \mathrm{kDa}$ dextran into the capsules (Figure 5).

\section{Discussion}

We studied optimization of encapsulation size by comparing the viability and insulin release of

A

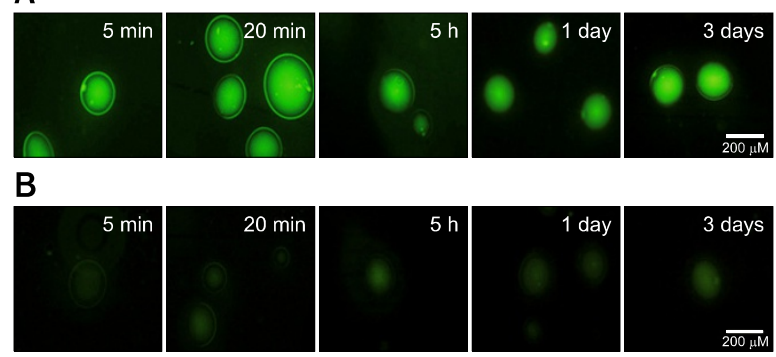

C

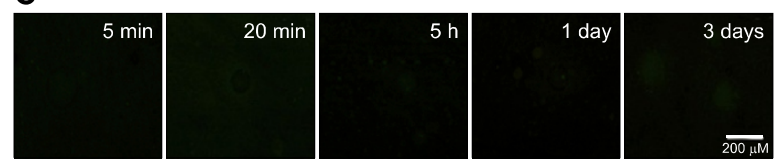

Figure 5. Fluorescence microscopy images of the calcium alginate capsules treated with 20 (A), 70 (B) and 150 (C) kDa FITC-dextran.

NPCCs in small and large capsules. Both small $(100-200 \mu \mathrm{m}$ in diameter) and large $(600-700 \mu \mathrm{m})$ capsules containing islets were produced using the air-driven droplet generating system.

Previous reports have suggested that the great distance between encapsulated islets and blood vessels would be one of the major causes of islet graft failure (de Groot et al., 2004). Since revascularization cannot occur within the capsule, the supply of nutrients and oxygen $\left(\mathrm{O}_{2}\right)$ to the islets is mediated only by passive diffusion instead of direct delivery of $\mathrm{O}_{2}$ from the blood to endovascular transplanted islets. Therefore, a fatal consequence of this large diffusion distance is the limited supply of oxygen, or hypoxia. $\mathrm{O}_{2}$ supply to the islets far from blood vessels is severely reduced because of the low aqueous solubility of $\mathrm{O}_{2}$, which induces islet dysfunction and necrosis after transplantation. A possible solution to hypoxia is reducing the diffusion distance between the islets and vessels through modification of the capsule size. Our results are in accord with this solution. The viability of NPCCs in small capsules was $44.6 \%$ higher than that of the NPCCs in large capsules after 28 days. In addition to this viability difference, the enhanced insulin secretion from the NPCCs in small capsules was observed, as shown in Figure $3 \mathrm{~B}$. Depletion of $\mathrm{O}_{2}$ supply through thick encapsulation within the large capsules promotes apoptosis and low insulin release. Both $\mathrm{O}_{2}$ supply and the delivery of glucose and insulin by passive diffusion are interfered with, causing a reduced insulin release in response to glucose stimulation (De Vos et al., 1996b).

Small capsules with a minimal amount of dead space would address the issue relating to total transplantation volume as well as oxygen supply. 
The diameter of the large capsules should be approximately three times as large as the original islets, increasing the islet suspension volume 27 times, which makes us find a site to implant such a large volume difficult (Teramura and Iwata, 2010). Therefore, we aimed to produce small capsules whose size is similar to NPCCs'. Alginate encapsulation was performed by changing encapsulatin conditions in order to control the size of produced capsules. Capsule size affects to flow rate of both alginate solution and air jacket (Wolters et al., 1991). In this research, only air pressure was controlled with constant alginate flow. Alginate flow rate was fixed as $135 \mathrm{ml} / \mathrm{h}$, the minimum value to avoid harsh shear force to islet and to show perfect spraying shape at injecting part of nozzle. At result, under the air pressure of 0.10 bar, capsules whose average diameter is $184( \pm 46.1) \mu \mathrm{m}$ was obtained. Finally, optimized encapsulation conditions using a $2 \%$ alginate solution were adapted to produce around $200 \mu \mathrm{m}$ alginate capsules.

However, a high percentage of irregular surfaces due to incomplete encapsulation induced pericapsular overgrowth and graft failure were observed with the small capsules (Halle et al., 1994; De Vos et al., 1996a). Although Calafiore et al. (1999) have reported small microcapsules of $300 \mu \mathrm{m}$ in diameter, the clinical application of the capsules resulted in large vessel plugging (Teramura and Iwata, 2010). Uniform islet dispersion in the alginate solution and air bubble elimination prevent not only islet protrusion from the capsule but also islet aggregation which disrupts alginate solution spraying because islets were isolated and space between islets is filled with alginate. Such a viscous alginate solution cover whole surface of islets. By optimization of encapsulation condition, we may give sufficient shear stress to alginate solution around islets not to wash-out attached alginate on islet surface. Consequently, alginate solution makes thin cover on islets without empty surface, which results in perfect small size encapsulation. In contrast to previous reports applying electrostatic encapsulation or a two-phase aqueous emulsification procedure (Halle et al., 1994; Calafiore et al., 1999), we successfully laminated NPCCs within small complete capsules (100-200 $\mu \mathrm{m})$ using an air-driven encapsulator without islet protrusion.

Interestingly, the viability and SI of naïve NPCCs at days 14 and 28 were significantly lower than those of the NPCCs encapsulated in small capsules, primarily due to a loss of interaction with the extracellular matrix (ECM). Islet-ECM interactions are known to be essential for the formation of islet structure (Montesano et al., 1983) and it has been suggested that the ECM may be implicated in islet survival and function (Wang and Rosenberg, 1999). The loss of contact with the ECM is associated with collagenase digestion during the isolation procedure. The phenomenon related to the ECM interaction loss is described by anoikis, which occurs in islets following disruption of interactions with the ECM. Frisch et al. (Frisch and Screaton, 2001) have indicated that protein kinase/phosphatase signaling molecules play a significant role in the regulation of anoikis through PI3K/AKT and raf/ERK. Additionally, it has been demonstrated that ECM interactions serve a role in the glucose responsive, $\beta$ cell (Hammar et al., 2005), which explains the low SI of the naïve NPCCs. Moreover, the naïve NPCCs are sensitive to physical stress and prone to be disintegrated during culture in vitro, as shown in Figure $1 \mathrm{~A}$. This stress is different for the encapsulated NPCCs, which are provided with structural support by alginate (Figures $1 \mathrm{~B}$ and 1C). Because physical shear stress may occur after transplantation in vivo, the alginate encapsulation of the islets may contribute to improving islet survival.

Our experimental results illustrated that the small capsules improved islet survival and insulin secretion ability when compared to the naïve NPCCs and NPCCs in large capsules. The capsules must possess selective permeability for nutrients with islet xenotransplantation for diabetes. Thus, there is a need to confirm that the capsule possesses permeability to molecules of low molecular weight such as glucose, nutrients, insulin and metabolites ( $<75 \mathrm{kDa})$. The immunoglobulin molecular weight limits are 150, 500 and $800 \mathrm{kDa}$ for $\mathrm{lgG}$, activated complements and IgM, respectively (Vériter et al., 2010). To this end, 100 to $200 \mu \mathrm{m}$ alginate capsules were used to confirm the penetration of variously sized dextrans. There was no penetration by $150 \mathrm{kDa}$ dextrans, whereas the 20 and $70 \mathrm{kDa}$ dextrans easily penetrated the small capsules. Indeed, the penetration of materials inside the capsule was not dependent on the thickness of the alginate capsule but rather on the pore size of the semi-permeable capsule, which can be controlled by the initial alginate solution concentration and alginate molecular weight (Schmidt et al., 2008). We confirmed that the small capsules produced by an air-driven encapsulator could effectively protect islets from immune cells and allow nutrient and metabolite transfer.

In conclusion, we optimized and evaluated capsule sizes for the encapsulation of NPCCs and found that the microencapsulation of NPCCs in the small capsules demonstrated significantly increased viability and insulin release capacity. Conditions for 
the optimal small capsule formation were established using an air-driven encapsulator. Moreover, the small capsules successfully demonstrated selective permeability, which includes the blockage of immune cells and penetration of insulin and glucose. Further studies regarding in vivo biocompatibility, glucose control and protection of islet cells in vivo using antioxidants or anti-inflammatory drugs (Zhu et al., 2010) should be conducted to provide a useful tool for treating diabetes mellitus patients.

\section{Methods}

\section{Materials}

All chemicals and reagents used in this study for isolation, culture and encapsulation of neonatal pancreatic cell clusters (NPCCs) in this study were purchased from SigmaAldrich Co. (St. Louis, MO), unless otherwise stated.

\section{NPCCs isolation}

One to three-day-old neonatal pigs were used as pancreatic donors. NPCCs were isolated according to previously established methods (Mazzitelli et al., 2008). Briefly, the piglets were intramuscularly administered with $0.1 \mathrm{mg} / \mathrm{kg}$ azaperon (stresnil, Janssen, Bruxelles, Belgium), 125 $\mathrm{mg} / \mathrm{kg}$ tiletamine hydrochloride and zolazepam hydrochloride (zoletil, Virbac, Carros, France), sequentially. The piglets underwent laparotomy to expose the pancreas by midline incision. The pancreas was then carefully dissected from its surrounding pylorus, duodenum and arteries. Particular care was administered to avoid bacterial contamination which is especially induced by bowel nicking. The pancreas was cut into small pieces $\left(2 \mathrm{~mm}^{3}\right)$ and washed in Hank's balanced salt solution (HBSS). The tissue was then digested using $2.5 \mathrm{mg} / \mathrm{ml}$ collagenase $\mathrm{V}$ for 7 to $10 \mathrm{~min}$ in a $37^{\circ} \mathrm{C}$ distilled water (DW) bath until its color appeared milk-like. The digested pellets were centrifuged at 1,000 rpm for $2 \mathrm{~min}$ and the pellets subsequently washed twice in HBSS supplemented with $100 \mathrm{U} / \mathrm{ml}$ penicillin, $0.1 \mathrm{mg}$ streptomycin (Invitrogen, Carlsbad), 0.25\% bovine serum albumin fraction $\mathrm{V}(\mathrm{BSA})$ and $12.5 \mathrm{mM}$ HEPES. Finally the tissue was resuspended in Hams-F10 supplemented with $0.5 \%$ BSA fraction $\mathrm{V}, 50 \mathrm{mM}$ 3-isobutyl-1-methylxanthine (IBMX), $10 \mathrm{mM}$ nicotinamide, $2 \mathrm{mM}$ L-glutamine, $100 \mathrm{U} / \mathrm{ml}$ penicillin and $0.1 \mathrm{mg} / \mathrm{ml}$ streptomycin and was plated in $100 \times 15 \mathrm{~mm}$ Petri dishes. The culture medium was changed every $48 \mathrm{~h}$.

\section{Optimizing encapsulation conditions}

Solubilized sodium alginate (Pronova UP-LVG, NovaMatrix, Sandvika, Norway) in saline as a concentration of 2 $\%(w / v)$ was transferred using a syringe pump (KDS100, KD Scientific Inc., Holliston) with a speed of $135 \mathrm{ml} / \mathrm{h}$, and the droplets were formed using an aerodynamic encapsulator (J30 encapsulator, Nisco Engineering AG, Zurich, Switzerland). The nozzle diameter was $500 \mu \mathrm{m}$. Spayed al- ginate droplets were hardened via crosslinking in $110 \mathrm{mM}$ $\mathrm{CaCl}_{2}$ solution for 5 to 10 minutes and washed twice with saline. Diameter of each capsules were measured by optical microscope.

\section{Encapsulation of NPCC using an air-driven encapsulator}

The NPCCs were cultured in vitro and then harvested by centrifugation and resuspended in a sodium alginate solution, resulting in a final concentration of $2 \%(\mathrm{w} / \mathrm{v})$ in saline. The alginate solution containing NPCC was gently pipetted several times until the islets were homogeneously dispersed, and the air bubbles inside the solution were removed by centrifugation. To investigate the effect of small $(100-200 \mu \mathrm{m})$ and large $(700-800 \mu \mathrm{m})$ capsules on the encapsulated NPCCs, both capsule types were prepared. For the small capsules, previous encapsulation method was applied under same conditions. For the large capsules, an air-driven encapsulating system with an alginate nozzle diameter of $500 \mu \mathrm{m}$ was designed, whose principle of entire system was similar to previous research (Wolters et al., 1991), was employed. Both droplets were hardened via crosslinking in $110 \mathrm{mM} \mathrm{CaCl}_{2}$ solution for $5 \mathrm{~min}$ and washed twice with saline.

\section{In vitro assessments of encapsulated NPCC}

After encapsulation, the NPCCs (naïve, small and large encapsulated groups) cultured in vitro were examined for viability and insulin release. Briefly, at days 1, 4, 7, 14, 21 and 28 of culture after encapsulation, the viability was evaluated by methylene blue staining using an automated cell counter (Invitrogen). The encapsulated NPCCs were also tested at days 14 and 28 for their insulin secretion in response to glucose stimulation. Selected NPCCs were sequentially incubated with 2.8 and $20 \mathrm{mM}$ glucose for $2 \mathrm{~h}$, respectively. The supernatants were retrieved at the end of each incubation period and stored at $-20^{\circ} \mathrm{C}$. The insulin content in the supernatants was measured using an electrochemiluminescence immunoassay (Elecsys insulin reagents kit, Roche Diagnostics, Mannheim, Germany) with an automated Roche Modular Analytics E170 (Roche Diagnostics). The stimulation index (SI) represents the capacity for insulin release and was calculated as the ratio of stimulated insulin to basal insulin (response to $20 \mathrm{mM}$ glucose/response to $2.8 \mathrm{mM}$ glucose).

\section{Permeability studies}

Approximately 100 alginate capsules were incubated with $0.1 \%$ fluorescein isothiocyanate-labeled dextran (FITCdextran) with molecular weights of 20,70 and $150 \mathrm{kDa}$ in DW. After $5 \mathrm{~min}, 20 \mathrm{~min}, 5 \mathrm{~h}, 1$ day and 3 days, the capsules were retrieved and washed three times with DW. Afterwards, the capsules were transferred to a microscope slide, promptly covered with a glass cover slip and then imaged under a fluorescence microscope (Nikon Eclipse TE2000-U, Tokyo, Japan). 


\section{Statistical analyses}

All data were subjected to one-way ANOVA, followed by Tukey's test using Prism version 4.0 (Graphpad Software, San Diego, CA) to determine the experimental group differences. The statistical significance was determined when the $P$-value was $<0.05$.

\section{Acknowledgements}

This study was supported by MKE (\#10033805, $\# 1033839$ ), BK 21 for Veterinary Science and Hanwha L\&C. The authors appreciate the TS Corporation for their supply of neonatal pigs, Youn Sang Kwon for transportation and Juho Hong and Hye-Jung Yeom for technical advice with NPCC isolation.

\section{References}

Calafiore R, Basta G, Luca G, Boselli C, Bufalari A, Cassarani MP, Giustozzi GM, Brunetti P. Transplantation of pancreatic islets contained in minimal volume microcapsules in diabetic high mammalians. Ann N Y Acad Sci 1999;875:219-32

de Groot M, Schuurs TA, van Schilfgaarde R. Causes of limited survival of microencapsulated pancreatic islet grafts. J Surg Res 2004;121:141-50

De Vos P, De Haan B, Pater J, Van Schilfgaarde R. Association between capsule diameter, adequacy of encapsulation, and survival of microencapsulated rat islet allografts. Transplantation 1996a;62:893-9

De Vos P, Vegter D, De Haan BJ, Strubbe JH, Bruggink JE, Van Schilfgaarde R. Kinetics of intraperitoneally infused insulin in rats. Functional implications for the bioartificial pancreas. Diabetes 1996b;45:1102-7

Frisch SM, Screaton RA. Anoikis mechanisms. Curr Opin Cell Biol 2001;13:555-62

Halle JP, Leblond FA, Pariseau JF, Jutras P, Brabant MJ, Lepage Y. Studies on small ( $<300$ microns) microcapsules: II--Parameters governing the production of alginate beads by high voltage electrostatic pulses. Cell Transplant 1994;3: 365-72
Hammar EB, Irminger JC, Rickenbach K, Parnaud G, Ribaux P, Bosco D, Rouiller DG, Halban PA. Activation of NF-kappaB by extracellular matrix is involved in spreading and glucosestimulated insulin secretion of pancreatic beta cells. J Biol Chem 2005;280:30630-7

Lim F, Sun AM. Microencapsulated islets as bioartificial endocrine pancreas. Science 1980;210:908-10

Mazzitelli S, Tosi A, Balestra C, Nastruzzi C, Luca G, Mancuso F, Calafiore R, Calvitti M. Production and characterization of alginate microcapsules produced by a vibrational encapsulation device. J Biomater Appl 2008;23: 123-45

Montesano R, Mouron P, Amherdt M, Orci L. Collagen matrix promotes reorganization of pancreatic endocrine cell monolayers into islet-like organoids. J Cell Biol 1983;97: 935-9

Schmidt JJ, Rowley J, Kong HJ. Hydrogels used for cell-based drug delivery. J Biomed Mater Res A 2008;87: 1113-22

Teramura Y, Iwata H. Bioartificial pancreas microencapsulation and conformal coating of islet of Langerhans. Adv Drug Deliv Rev 2010;62:827-40

Vériter S, Mergen J, Goebbels RM, Aouassar N, Gregoire C, Jordan B, Leveque P, Gallez B, Gianello P, Dufrane D. In vivo selection of biocompatible alginates for islet encapsulation and subcutaneous transplantation. Tissue Eng Part A 2010; 16:1503-13

Wang RN, Rosenberg L. Maintenance of beta-cell function and survival following islet isolation requires re-establishment of the islet-matrix relationship. J Endocrinol 1999; 163:181-90

Wolters GH, Fritschy WM, Gerrits D, van Schilfgaarde R. A versatile alginate droplet generator applicable for microencapsulation of pancreatic islets. J Appl Biomater 1991;3: 281-6

Zhu H, Wang J, Jiang H, Ma Y, Pan S, Reddy S, Sun X. Bilirubin protects grafts against nonspecific inflammationinduced injury in syngeneic intraportal islet transplantation. Exp Mol Med 2010;42:739-48 